verschwinden mehr und mehr, und Eisenbeton ist längst fast das einzige Baumaterial im Lande geworden. Bäume an den Straßen entlang gibt es nicht, aber in den vielen Gärten um die Häuser in der Stadt, weitgehend auch in der Innenstadt, wachsen hübsche Ebereschen und vielfach jetzt auch immergrüne Sitkatannen, die aus Alaska eingeführt worden sind und in dem isländischen Klima anscheinend ausgezeichnet gedeihen. In manchen Gärten herrscht eine Blumenfülle von beinahe südlicher Leuchtkraft.

Trotz allem liegt über dieser gesetzlosen Unordnung und schreienden Farbenpracht, welche die junge Hauptstadt des Landes kennzeichnen, ein gewisser Charme, der sich schwer erklären läßt. Sicher trägt die klare nordische Luft, die keine rauchenden Schornsteine kennt, ihren Teil dazu bei, wie auch der isländische Himmel mit seinen Wolkenbildungen, welche den Häusern der Stadt an Vielfältigkeit der Formen und Farben nichts nachgeben, die Berge in der Runde und schließlich die weite Bucht.

Es ist wirklich schön in Reykjavik an schönen Sommertagen, aber am schönsten ist es doch an stillen Frühlingsabenden, wenn der Himmel sich im schattigen Mantel des Sees spiegelt und die untergehende Sonne ihre Strahlenflut über das Meer ergießt, wenn die Eiskappe des Snæfellsjökull rosig gefärbt ist, wenn über der Esja goldgeränderte Wolken schweben und die Berge Akrafjall und Skardsheidi wie «veilchenfarbige Träume» sind. An solchen Abenden herrscht noch immer die gleiche Schönheit in Reykjavik wie vor tausend Jahren, jene Schönheit, die dem weisen Gesetzsprecher, Ingólfs Enkel, vor Augen schwebte, als er sich «in seiner Todeskrankheit in die Sonnenstrahlen hinaustragen lie $\beta$ und sich dem Gott anheimgab, der die Sonne geschaffen hatte».

Mit Kürzungen dem Buch «Island» (Verlag Kümmerly \& Frey) entnommen. Aus dem Isländischen übersetzt von Ute Jacobshagen.

\title{
DER ST. LORENZ-SEEWEG \\ PLANUNG UND GESTALTUNG EINER STROMLANDSCHAFT
}

\section{ERNST WinkLER}

Im Beisein Elisabeths II von England und Präsident Dwight Eisenhowers wurde am 26. Juni 1959 der St. Lorenz-Seeweg eröffnet, der die großen Seen Nordamerikas mit dem atlantischen Ozean verknüpft. «Seit den Tagen», sagte die Königin in ihrer Festrede, «da sich die unerschrockenen französischen Entdecker und Siedler an den Ufern dieses Flusses niederließen, träumten die Menschen von einem Schiffahrtsweg vom Hafen von Montreal bis zu den Großen Seen. Mehrere Generationen von französisch- und englisch-sprechenden Kanadiern haben an der Verwirklichung dieses grandiosen Projektes gearbeitet. Es ist daher angebracht, daß wir den Weitblick der Männer preisen, die als erste das Projekt geplant haben. Aber wir müssen auch den Mut und den Weitblick der Politiker der beiden Länder anerkennen, die die für die Verwirklichung des Projektes benötigte politische Übereinstimmung erleichterten». Präsident Eisenhower sekundierte, indem er betonte: «Der St. Lorenz-Schiffahrtsweg, der die Weltmeere mit den Großen Seen des nordamerikanischen Kontinents verbindet, ist die Verwirklichung der Träume von Tausenden von Menschen auf beiden Seiten der kanadisch-amerikanischen Grenze».

Mit dieser Einweihung, der die Freigabe des Kanals für den Schiffsverkehr schon am 25. April des gleichen Jahres vorausgegangen war, hatte in der T'at ein Werk Abschluß gefunden, das seit Jahrzehnten, wenn nicht seit Jahrhunderten ersehnt und gewünscht worden war. Die fast vierzig Jahre umfassende Vorgeschichte der eigentlichen Nutzbarmachung des St. Lorenzstroms für Ozeanschiffe und als Energielieferant hatte allerdings keineswegs durchwegs den 


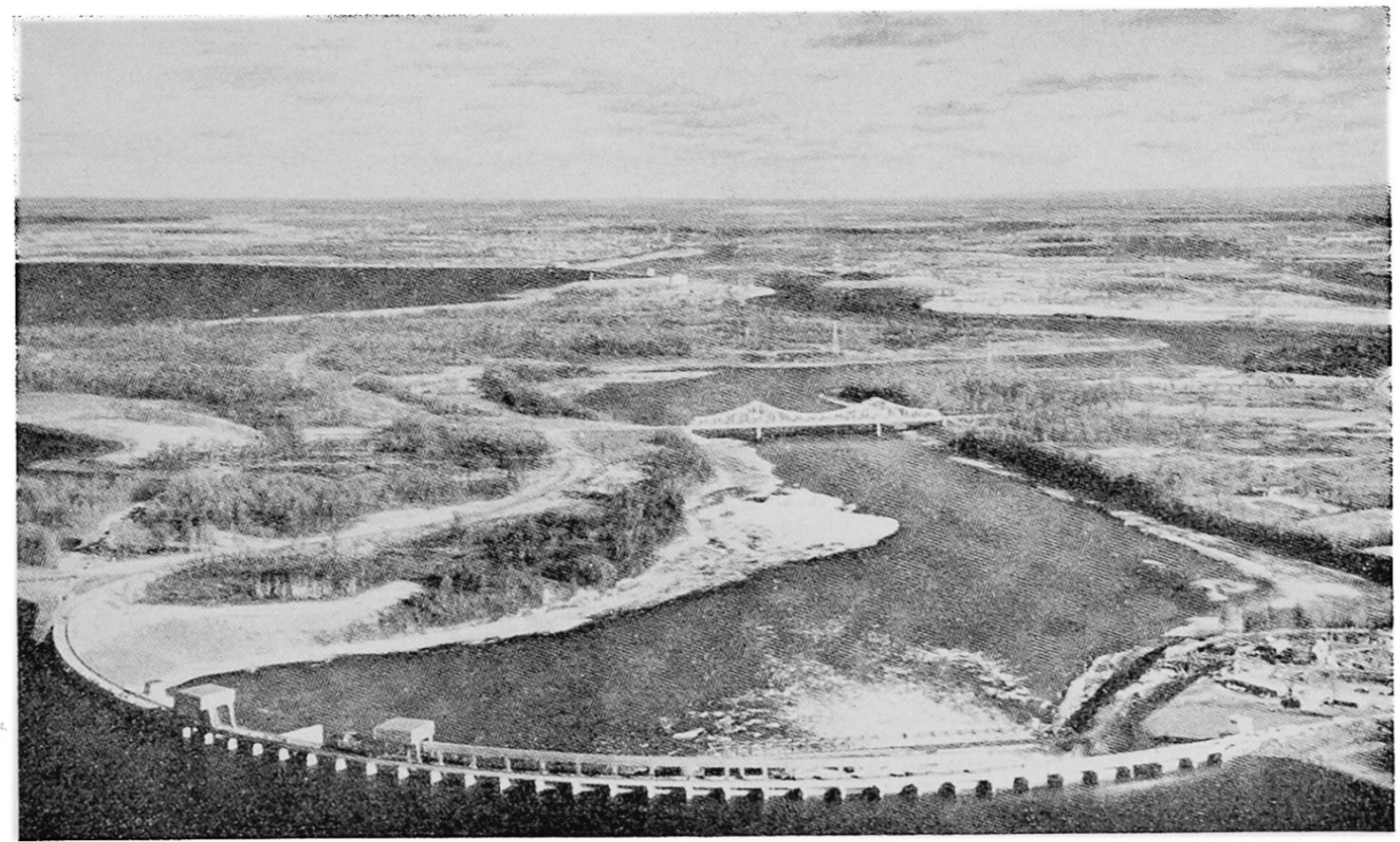

Die Landschaft des St. Lorenz-Stroms. Im Vordergrund der Bogen des Long Sault Dammes längs des südlichen Kanalabschnitts. Im Hintergrund der St. Lorenzkraftwerkdamm und (links) die Stadt Cornwall.

Geist erkennen lassen, den die beiden Landeshäupter anläßlich der Einweihung beschworen. Diese Vorgeschichte stand vielmehr lange im Zeichen der Gegnerschaft der Vereinigten Staaten von Nordamerika, die diese in einem wenig positiven Lichte zu sehen nötigt. Zwar hatte deren Regierung seit langem die Einsicht gewonnen, daß der Kanalbau durchaus auch im Interesse des eigenen Landes liege. Sie hatten deshalb bereits $19+1$ mit Kanada einen Ausbau-Vertrag abgeschlossen und den Kongre $\beta$ zu dessen Ratifizierung angehalten; allein von letzterem war der Verwirklichung hartnäckigen Widerstand entgegengesetzt worden, weil er unter dem Druck verschiedener Wirtschaftsmächte stand, die in einer neuen Wasserstraße und einem neuen Kraftwerk eine Bedrohung ihrer eigenen Interessen erblickten. Insbesondere hatten die Eisenbahngesellschaften eine Abwanderung vieler Gütertransporte (so Getreide) auf den billigeren Wasserweg befürchtet, und aus analogen Motiven stemmten sich die Stadt New York und andere Hafenorte der Ostküste dem Projekt entgegen. Die Erdölindustrie und der Kohlenbergbau wiederum wie auch die privaten Elektrizitätswerke bangten vor der Konkurrenz billigen Stromes und hatten sich mit jenen sogar zu einer gemcinsamen Organisation, der «National St. Lawrence Project Conference» zusammengeschlossen, deren einziges Ziel die Verhinderung der Projektrealisierung bildete. Die andauernde Verschleppung der Frage durch den amerikanischen Kongreß erschöpfte endlich die Langmütigkeit der Kanadier. Ihre Regierung beschloß im Herbst 1951, nicht zuletzt auf Grund des im zweiten Weltkrieg gewonnenen Bewußtseins einer entscheidenden finanziellen Machtzunahme des Landes, den neuen St. Lorenz-Kanal allein zu bauen. Dadurch wäre er nach den damals entworfenen Plänen so gut wie ganz auf die kanadische Seite des St. Lorenz zu liegen gekommen, was technisch auch durchaus möglich erschien. Die einstimmige Verabschiedung des Gesetzes durch das Parlament in Ottawa im Dezember des gleichen Jahres beeindruckte Washington entschieden. Als Kanada, gebeten noch etwas zuzuwarten, abschlägig antwortete, erteilte die Federation Power Commission Ende 1953 auf Weisung der Regierung plötzlich die Konzession zum Bau der amerikanischen Kraftwerkhälfte an die Power Authority des Staates New York, und anfangs $195+$ gelang den Senatoren Wily und Dondero die Opposition zu überwinden, so daß Präsident Eisenhower am 13. Mai das Gesetz über die Gründung der amerikanischen Saint Lawrence Seaway Development Corporation unterzeichnen konnte. Daraufhin lenkten auch die Kanadier wieder ein und die gemeinsame Verwirklichung der Kanalisation und der Kraftwerkanlage konnte in Angriff genommen werden. Die amerikanische Schwenkung in der Frage hatten keineswegs allein Prestigerücksichten wie etwa die Überlegung bestimmt, daß man Kanada nicht allein das unbedingt historische Werk ausführen lassen könne. Ebensosehr waren es rein wirtschaftliche Argumente: die Einflußnahme auf die Tarifgestaltung der Flußschiffahrt, die Versorgung mit Eisenerzen aus Labrador u. a., welche nunmehr auch die Amerikaner dazu brachten, die Errichtung des Seeweges als wirtschaftlich dringlich wie auch im Interesse der nationalen Sicherung für not- 
wendig anzusehen. So wurden denn im Spätsommer 1954 die Bauarbeiten aufgenommen, nachdem vorher für die Ausnutzung der Hydroenergie wie für den Seeweg die verantwortlichen Organisationen geschaffen und alle rechtlichen Fragen gelöst waren. Die «vierte Küste»Amerikas konnte endlich geschaffen werden.

Das in der Folge in der kurzen Zeitspanne von bloß fünf Jahren vollendete Werk kann nur aus der Natur und der Geschichte des nordamerikanischen Kontinents verstanden werden. Es sei deshalb im folgenden versucht, sie knapp zu umreißen.

Als Ausgangspunkt darf wohl die Tatsache hervorgehoben werden, daß das sogenannte Seen-St. Lorenz-Gebiet zwar nur etwa einen Prozent der Gesamtfläche Kanadas (das Einzugsgebiet des St. Lorenzstroms macht immerhin mit rund 1,8 Millionen $\mathrm{km}^{2}$ über $10 \%$ aus) beträgt, daß aber in diesem Gebiet zwischen $60-70 \%$ der gegenwärtig (1960) nahezu 18 Millionen kanadischen Bevölkerung leben. Die Region muß also offenbar eine entscheidende Anziehungskraft auf die Besiedler des Landes ausgeübt haben, die selbst diejenige der Ostabdachung der Vereinigten Staaten, wo sich ja gleichfalls ein großer Prozentsatz ihrer Bevölkerung konzentriert, übertrifft. Wohl die Hauptgründe dieser jedenfalls für Kanada einzigartigen Bevölkerungsballung liegen in Lage und Natur beschlossen, wobei freilich kaum leicht abzuschätzen ist, welchem unter ihnen der Vorrang gebührt. Sicher aber hat die Lagerung der Seen-St. Lorenztalung zwischen den drei Hauptregionen des nordamerikanischen Kontinents: zwischen den Appalachen, den großen Ebenen des Innern (Great oder Interior Plains oder Lowlands) und dem Kanadischen Schild (dem Shield oder auch Laurentian Plateau), die zugleich enge Verklammerung dieser Gegenden bedeutet, an der Entwicklung zum volksreichsten Gebiet und «Herzland» Kanadas maßgeblichen Anteil, ebenso wie dessen relative Nähe zum europäischen Kontinent (Schiffsroute Liverpool-Quebec $4235 \mathrm{~km}$ -Montreal $4448 \mathrm{~km}$ - New York $4971 \mathrm{~km}$ ) bei der Erschließung eine wesentliche Rolle gespielt hat. Daß hierbei ein Strom vom Charakter des St. Lorenz, der, indem er gewissermaßen ins Zentrum des Kontinents hineinführt und nach Ansicht der Amerikaner dessen Küsten um nahezu $13000 \mathrm{~km}$, d. h. um mehr als die ganze atlantische Front Nordamerikas, verlängert, von entscheidender Bedeutung werden mußte, braucht kaum betont zu werden. Hält man dazu die weitere Tatsache, daß die natürliche Ausstattung des Gebietes: vor allem Boden und Klima die menschliche Niederlassung ebenfalls begünstigen, wenn auch Härten und Hemmnisse keineswegs fehlen, dann wird die erwähnte Bevorzugung der St. Lorenztalung durch Siedlung und Wirtschaft evident. Die so umrissene Natureigenart ist selbstredend ein Ereignis sehr langer erdgeschichtlicher Entwicklung. An ihrem Anfang steht die Auffaltung und der nachfolgende Abtrag, die Einebnung der Nordhälfte des nordamerikanischen Erdteils, die ins Präkambrium, ja an dessen Ausgangsepochen: das Archäozoikum zurückzudatieren ist. Ihm wurde in der Folge - als Ergebnis des Gebirgsabtrags - weite Sedimentationsgebiete vorgelagert, von denen weiterhin offensichtlich nur die südöstliche Randzone (permisch) stärker gefaltet worden ist, die ihrerseits selbst wieder wesentlich eingeebnet, d. h. durch Erosion erniedrigt wurde. Wie weit der Faltenwurf dieser heute Appalachen genannten Großregion die Grabenbildung zwischen ihr und dem Kanadischen Schild mitbestimmte, bleibt ferneren Untersuchungen vorbehalten. Jedenfalls folgt der St. Lorenzstrom mindestens in seiner unteren Laufstrecke einer alten Störung, deren Alter mit demjenigen der Appalachen weitgehend übereinstimmt, und den Südteil, zwischen Quebec und den Adirondacks, markieren so deutliche, bis $800 \mathrm{~m}$ hohe Steilabfälle zum Senkungsfeld der Talung, daß Brüche bzw. Verbiegungen der ältern, vorpleistozänen Erdrindenoberfläche bei deren Bildung kaum zu bezweifeln sind, wenn auch Sedimentations- und Erosionsvorgänge die Hauptrolle gespielt haben mögen. Maßgebend für das quartär-alluviale Relief wurde jedoch die pleistozäne Vergletscherung, die vor allem das Entwässerungssystem, d. h. die Richtung der Täler im Bereich der nachmaligen Großen Seen und des St. Lorenz nachhaltig beeinflußte. Während der Vereisung waren die Schmelzwasser genötigt, zur Hauptsache nach Süden, gegen den 
Die Hauptabschnitte des neuen Seeweges (Nach V. Scott)

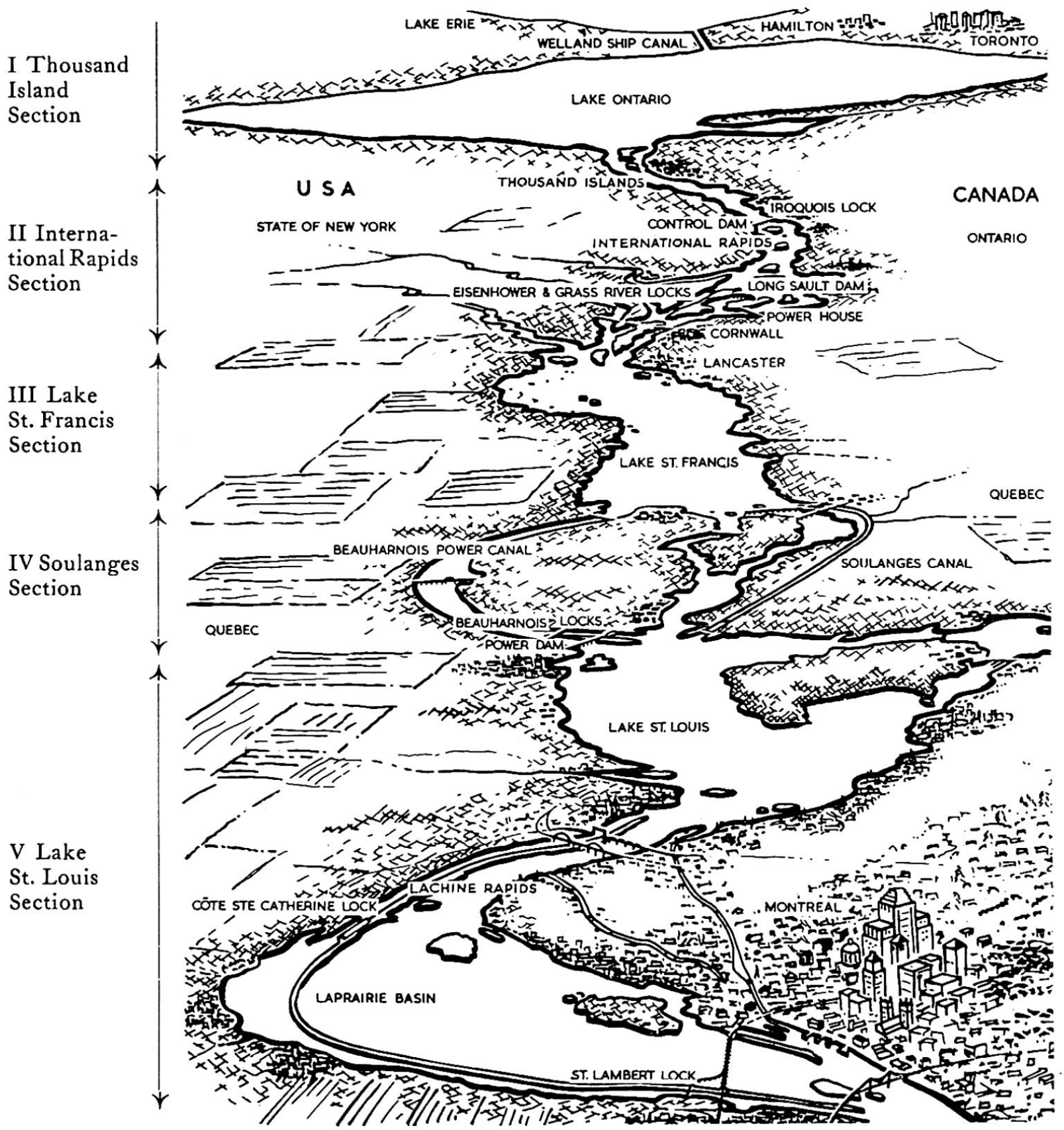

Golf von Mexiko, abzufließen. Der Rückzug der Gletscher dagegen führte im $\mathrm{Zu}$ sammenhang mit Landhebungen als Folge isostatischer Ausgleichsbewegungen seit etwa 20000 v. Chr. zu einer Neuorientierung der Flußrichtungen. Das Endergebnis war die Ausbildung des hydrographischen Systems der Großen Seen $\left(247400 \mathrm{~km}^{2}\right.$ ), die nunmehr nach Nordosten, durch die Senke des St. Lorenz, ihren Abfluß fanden. So entwässert der Obere See (Lake Superior 183,5 m ü. M.) durch die Enge von Sault Sainte Marie zur Seengruppe des Lake Michigan und Lake Huron mit der Georgian Bay (176,0 m ü. M.). Sie ist durch den Detroit und St. Clair River mit dem nur wenig tiefer $(174,3 \mathrm{~m})$ gelegenen Erie-See (Lake Erie) verknüpft, von welchem aus der Niagara River mit den gleichnamigen weltbekannten Fällen (48-60 m) die Verbindung mit dem wesentlich tiefer gelegenen Ontario-See (75 m ü. M.) herstellt. Dessen Abfluß, der St. Lorenzstrom hat zunächst, bis Montreal (25-60 m ü. M.) eine rund $250 \mathrm{~km}$ lange sehr schnellenreiche Strecke zu überwinden, dann aber fließt er in 
ruhigem Lauf nach Nordosten, um nach abermals etwa $250 \mathrm{~km}$, ab Quebec ein $400 \mathrm{~km}$ langes und 10-50 km breites Aestuar zu bilden und schließlich in den $210000 \mathrm{~km}^{2}$ großen St. Lorenz-Golf zu münden. Bemerkenswert ist, daß ihm auf dieser Gesamtstrecke, ebenso wie den Seen, nur wenige größere Flüsse tributär sind, so der oberhalb Montreal mündende Ottawa River $(914 \mathrm{~km})$ und der nördlich Quebec mündende Saguenay $(760 \mathrm{~km})$ beide von links kommend. Nicht zuletzt diesem Umstand, aber wohl vor allem dem großen Anteil von Seen (nahezu 15\% des Einzugsgebietes), die als imposante Retensionsbecken funktionieren, verdankt der Strom die relativ geringen jahreszeitlichen Wasserstandsschwankungen (1860-1940 im Mittel zwischen 4078 und $8892 \mathrm{~m}^{3} / \mathrm{sec}$; Jahresmittel $6712 \mathrm{~m}^{3} / \mathrm{sec}$ ), die grundsätzlich $1: 2$ betragen, während beim Mississippi ein Verhältnis von 1:25, beim Columbia River 1:35 besteht. Dieses ausgeglichene Abflußregime zusammen mit den verhältnismäßig großen Wassermassen und der im ganzen genügend tiefen Fahrrinne war es vornehmlich, welches den St. Lorenz zum bedeutenden Verkehrsweg (und zugleich zur wichtigen Kraftquelle) prädestiniert hat, wenn auch vier- bis fünfmonatige Vereisung erhebliche Beeinträchtigungen bedingt.

Das nacheiszeitliche Relief dieser Fluß- und Seenlandschaft kennzeichnet im übrigen, von der flachen und breiten Stromtalung (in welcher allerdings an verschiedenen Stellen, z. B. bei Montreal, vereinzelte Inselberge (Monteregian Hills wie Mount Royal, Beloeil oder Yamaska) an die starke orogenetische Aktivität des Devons erinnern) und von den diese begleitenden flachen Plateaus (der Laurentiden im Westen 300-400 m, der Appalachen im Osten 800-1500 m) abgesehen die ganze Mannigfaltigkeit glazialer und subglazialer Kleinformen: Grund-, Seiten- und Endmoränen, Zungenbecken, Sandrebenen, die zudem mit alluvialen in weiten Terrassen zum Ausdruck kommenden Sedimentations- und Abtragungsvorgängen kombiniert ist. Für ihre Eignung als menschlicher Lebensraum war die Lage der Gesamtlandschaft zum Erdteil bzw. Atlantik entscheidend. Dabei überwiegen wohl die Einflüsse der riesigen Kontinentalmasse des Nordens (bzw. Westens) und der benachbart fließenden kalten Meeresströmungen (Labradorstrom), wenn andrerseits namentlich das St. Lorenztal auf Grund seiner Kontakte mit der Seenregion eine Wärmeinsel darstellt, die zudem teilweise noch den Regenschatten der Appalachen genießt. Der weiten Nordsüderstreckung des Gebietes entspricht naturgemäß eine analoge klimatische Differenzierung in dieser Richtung, die sich im Wechsel der Pflanzendecke spiegelt.

Klimatabelle mit europäischen Vergleichsstationen

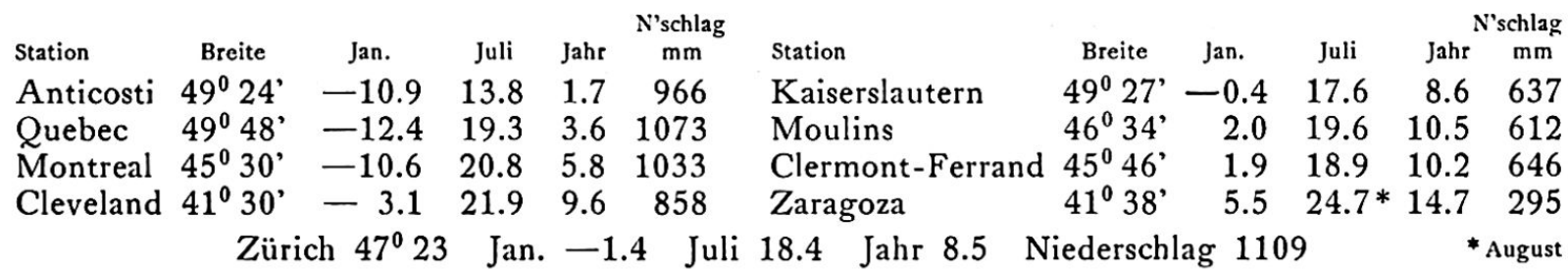

Der Unterschied der nordamerikanischen gegenüber den europäischen Stationen ist umso ausgeprägter als diese meist erheblich höher gelegen sind als jene; gegenüber den europäischen erweisen sich die kanadischen deutlich «überkaltet».

Den Süden wie die Halbinsel Ontario bedeckten ursprünglich zur Hauptsache Laubwälder, in denen der Zuckerahorn eine Leitpflanze bildete, wobei auf sandigen Stellen Kiefer-, besonders Rot- und Weymuthskieferbestände vorherrschten. Gegen Nordosten mischten sich verschiedene Eichenarten, der rote Ahorn, Linden, Eschen und Birken bei, in den Flußniederungen fanden sich Grasländer und Sümpfe. Nördlich Quebec ging die Vegetation allmählich in Nadelwälder über, in welchen Fichten, Rottannen und Balsamtannen neben Birken die Dominanten waren. Diese vornehmlichen Waldlandschaften waren noch zur Zeit der europäischen Invasion (und sind es teils noch 
jetzt) reich an Wild (Elch, Virginiahirsch, Schwarzbär, Wolf, wertvolle Pelztiere wie Feh, Skunk, Biber, zahlreiche Vogelarten usw.). Zudem bestanden seit langem Siedlungen verschiedener Indianerstämme, so daß mindestens lokal sich auch Kulturlandschaften ausgebildet hatten. Von Norden her waren nomadische Jäger, Fischer und Sammler bis ins St. Lorenztal hinein eingesickert; von Süden her hatten sich umgekehrt ackerbauende Irokesen durch die Champlain-Senke angesiedelt. Das eigentliche Tal selbst war wohl von Algonkins bewohnt, die vereinzelte palisadenbewehrte Dörfer wie Hochelaga auf der spätern Insel Montreal besassen. Die letztere hatte offenbar schon strategische Bedeutung, indem sie die Portage (den Tragplatz) bildete, der die LachineFälle umging und damit den gesamten Kanuverkehr am obern St. Lorenz und am Ottawafluß kontrollierte. Im Bereich der östlichen Großen Seen siedelten Huronen (an der Georgian Bay in etwa 30-40 Dörfern) und Irokesen, die trotz ihrer nahen völkischen Verwandtschaft verfeindet lebten. Doch durtfe wohl der Einfluß der Indianer auf die Landschaft im ganzen gering gewesen sein.

Der Wandel zur «kanadischen» Kulturlandschaft setzte ein, als nach tastenden Landeversuchen normannischer und bretonischer Fischer zu Beginn des 16. Jahrhunderts und nach der Besitzergreifung der Küsten durch den in französischen Diensten segelnden Italiener Verrazano, der Bretone Jacques Cartier auf seiner zweiten Entdeckungsreise 1535 den St. Lorenzgolf entdeckt hatte und an der Indianersiedlung Stadacona (jetzt Quebec) vorbei bis zu den großen Stromschnellen bei Hochelaga (Montreal) vorgedrungen war, die er, weil er ausgesandt war, China zu finden, Lachine nannte. Anfängliche Versuche der Ansiedlung französischer Kolonisten (um 1540-1550) scheiterten, so daß der Beginn der Kolonisation ins Jahr 1608 mit der Gründung von Quebec durch zu datieren ist. In der Folge entstanden mit der Anlage von Trois Rivières 1634, Montreal 1641 und Fort Richelieu 1642 die Ansatzpunkte zur eigentlichen Inwertsetzung des Landes, zur Entstehung von «Kanada» (nach dem Algonkinwort kanatta $=$ Niederlassung), das die Engländer später als Unterkanada (Lower Canada) im Gegensatz zum östlichen Seengebiet (das Oberkanada «Upper Canada» genannt wurde) bezeichneten. Während sich in der Folge bis 1763 eine ausgesprochen französisch anmutende Kulturlandschaft entfaltete, in welcher um 1700 rund 12000 , Mitte des 18. Jahrhunderts etwa 65000 Frankokanadier lebten, begann von den Küsten der mittlern und nördlichen Appalachen her eine zweite, englische Einwandererwelle, die dank ihrer viel größern Individuenzahl das französische Kolonialgebiet bald so sehr einengte, daß es auch militärisch den Briten unterlag. Seit 1763 (seit dem Frieden von Paris, der ganz Kanada England überantwortete) entwickelte sich das St. Lorenz-Seengebiet deshalb als Kernland der britischen Kolonisation, wenn auch den französischen Siedlern sowohl die Beibehaltung ihrer Muttersprache als auch der angestammten katholischen Religion zugestanden wurde. Diese Entwicklung steht im Zeichen einer bedeutenden Expansion der Wirtschaft. Deren Fundamente bilden bis heute der Wald als Holz-, Pelz- und Früchtelieferant, die Fischerei und die Bodenkultur (vor allem in Mischfarmbetrieb, im Norden mit ausgesprochener Viehzucht im Süden mit Obst-, Gemüse und Weinbau), während die Wasserkraftnutzung und der Bergbau (in den Randgebieten und längs den Großen Seen) erst sekundär hinzutraten. Noch anno 1800 besaß die Region jedoch kaum 500000 Einwohner. Diese Zahl stieg indes bis 1850 auf rund 2000000 , bis 1900 auf 3831000 , bis 1950 auf 8652 000, um Ende 1959 rund 10500000 zu erreichen. Damit hatte das Gebiet bis in die jüngste Gegenwart hinein den überragenden Anteil an der Gesamtbevölkerung Kanadas anzuziehen vermocht, wenn dieser auch von nahe $100 \%$ ums Jahr 1800 über 74 auf $63 \%$ gesunken ist und die mittlere Volksdichte kaum 5 Einwohner $\mathrm{km}^{2}$ (in der Talung selbst allerdings 50-200) beträgt. Im gleichen Zeitraum vollzog sich übrigens ein deutlicher Urbanisationsprozeß. Zwar lebte in den Anfängen der Besiedlung die Bevölkerung bereits in befestigten und damit vorwiegend stadtähnlichen Ortschaften 


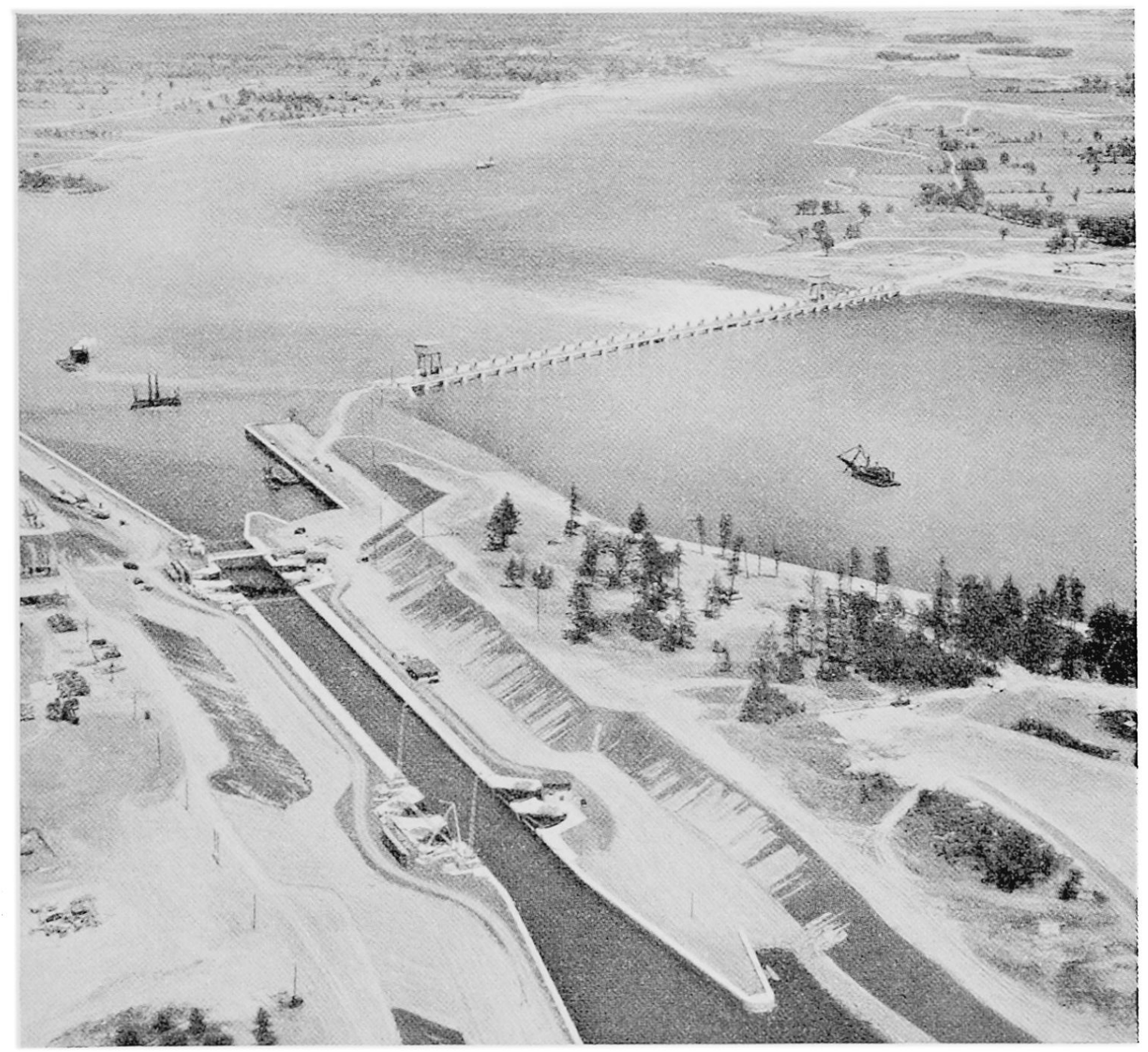

Die Iroquois-Schleuse. Sie leitet die Schiffe um den im Mittelgrund sichtbaren Iroquois-Damm. Links im Hintergrund das neue Dorf Iroquois. In der Umgebung sind zwei neue Dörfer im Entstehen begriffen.

konzentriert, in der Folge aber verteilte sie sich auf das Land. Doch die Industrialisierung im 19. Jahrhundert rief erneut einer Konzentration. Sie hatte allerdings noch um 1870 kaum 20\%, um 1900 dagegen schon 50\%, 1950 gegen 68\% der Bevölkerung der Gesamtregion erfaßt und dürfte gegenwärtig mehr als $70 \%$ betragen. An dieser Verlagerung nahmen einige wenige Städte, vor allem Montreal, die größte Stadt Kanadas, Toronto, Quebec, Ottawa, Hamilton und Windsor den Hauptanteil. Mit insgesamt 4,5 Millionen Einwohnern beanspruchten sie 1959 28\% der Bevölkerung ganz Kanadas und 43\% derjenigen der Region (Provinzen Quebec und Ontario).

Daß eine solche Bevölkerungsmassierung nur durch entsprechende wirtschaftliche Kapazität zustande kommen konnte, beleuchtet beispielhaft das Arbeitseinkommen Kanadas. Es wurde, sich allein zwischen 1950 bis 1958 von 10 auf 16,2 Milliarden Dollars steigernd, zu 65-70\% in der Region erworben. In der Güterproduktion führte gleichzeitig diese mit $60-65 \%$ der gesamtkanadischen. Ähnlich würden Angaben über Landwirtschaft und Industrieproduktion lauten, doch sei aus Raummangel auf ihre Nennung verzichtet. Es dürfte hieraus zur Genüge klar werden, daß die St. Lorenz-Seentalung mit Grund das «Herzland» Kanadas genannt wird, wenn auch in Zukunft mit ihr andere Gegenden wie der Mittelwesten und vielleicht British Colum- 
bia erfolgreich in Wettbewerb treten werden. Klar resultiert hieraus, daß diese Stellung nur mit der Ausnützung aller Möglichkeiten zu erzielen war und erhalten werden kann. In diesem Rahmen gewinnt die Schaffung und Verbesserung eines Seeweges durch den St. Lorenz vom Atlantik zur Kontinentmitte naturgemäß erneutes Gewicht und läßt sich erst eigentlich durch ihn verstehen. Zweifellos bedeutet das Werk nur ein Element der Inwertsetzung der ganzen Landschaft, aber immerhin ein bemerkenswertes und umso wichtigeres, als die Zukunftsentwicklung des ganzen Landes je länger desto stärker sich mit der übrigen Welt verflechten wird. In diese Entwicklung ist ein Strom von der Einmaligkeit des St. Lorenz - als Schiffahrtsweg wie als Kraftspender - notwendigerweise einzuschalten. Die Einsicht in seine Notwendigkeit nun stammt, wie schon eingangs angedeutet wurde, keineswegs von heute. Die Idee einer Kanalisation des St. Lorenzstromes, seine Ausgestaltung zur kontinentalen Verkehrsstraße reicht vielmehr in die Anfänge der Europäisierung Nordamerikas zurück. In ihrer ursprünglichen Konzeption mag sie mit dem Vordringen Samuel de Champlains in das Gebiet der Großen Seen verknüpft sein, also in die Jahre um 1609-1615 datieren, da Champlain als erster Europäer dem Lauf des St. Lorenz folgend bis zum Nipissingsee und zur Georgian Bay (Huronsee) gelangte. Er hatte schon 1599 versucht, Heinrich IV. von Frankreich zu einem Kanalbau durch die Landenge von Panama zu bewegen «der den Weg nach der Südsee um mehr als 1500 Meilen verkürzen würde». Den Bau eines Kanals dagegen scheint als erster Dollier de Casson, ein Prior des Sulpicienklosters in Montreal ums Jahr 1680 ins Auge gefaßt zu haben, als er vorschlug, eine Umgehungsrinne bei den Lachine-Fällen zu konstruieren. Damit begann eine nicht mehr unterbrochene Kette von Bauunternehmungen, die schließlich im vergangenen Jahr mit der Schiffbarmachung für Ozeandampfer gekrönt wurde.

H. Boesch schildert diese Geschichte ebenso knapp wie treffend: «Schon um 1700 wurde ein kleiner Kanal von nur $45 \mathrm{~cm}$ Tiefe zur Umgehung der Stromschnellen bei Lachine gegraben. 1780 und 1804 wurde dieser Vorläufer so verbessert, daß die großen canots de maitre einen Zugang zum Westen fanden. Zwischen 1843 und 1848 wurde dann der eigentliche Lachine-Kanal zur Úmgehung der Stromschnellen oberhalb Montreal vorerst mit 2,74 m, später (1870-1883) mit 4,27 m (14 Fuß) Tiefe gebaut und so die $15 \mathrm{~m}$ Höhendifferenz zum Lake St. Louis (Einmündung des Ottawa River) überwunden. Von hier aufwärts bis Lake St. Francis, einer Ausweitung im St. Lawrence, wurden die zahlreichen Schnellen schon in den Jahren 1779 bis 1783 mit kurzen Seitenkanälen von $75 \mathrm{~cm}$ Tiefe umgangen. Hierauf wurde vorerst auf der Südseite des Flusses in den Jahren 1842-1845 ein Vorläufer des heutigen BeauharnoisKanals $(2,74 \mathrm{~m})$, später (1892-1899) auf der Nordseite der Soulanges-Kanal $(4,27 \mathrm{~m}$ mit fünf Schleusen zur Überwindung der fast $26 \mathrm{~m}$ Höhendifferenz) konstruiert. Stromaufwärts folgten eine Reihe von weiteren Kanälen in der sogenannten International Rapids Section (so benannt, weil in diesem Abschnitt der St. Lawrence die Grenze zwischen den Vereinigten Staaten und Kanada bildet), die fast alle im vergangenen Jahrhundert gebaut wurden. Es sind dies: Cornwall-Kanal, Farrans's-PointKanal, Rapid-Plat-Kanal bei Morrisburg und Galop-Kanal bei Iroquois. Bis 1908 waren alle diese Kanäle auf 4,27 m ausgebaut worden. Das nächste bedeutende Hindernis, die Niagara-Stufe, wurde von allem Anfang an ganz auf kanadischem Gebiet durch einen Kanal umgangen (Welland-Kanal), der etwa $15 \mathrm{~km}$ westlich vom Niagara River verläuft. Ein erster Kanal wurde hier schon in den Jahren 1824-1833 gebaut und 1842-1850 auf 2,74 m vertieft. 1887 war der folgende Ausbau auf 4,27 m mit 26 Schleusen abgeschlossen, so daß 1908 nach der Fertigstellung der Kanäle unterhalb von Lake Ontario ein durchgehender Verkehr bis zu diesem Tiefgang zwischen dem Ozean und den Great Lakes möglich war. Inzwischen hatten aber die Great Lakes als Binnenwasserstraße eine immer größere Bedeutung erlangt. Es ist hier vor allem an die Erztransporte vom Lake Superior zum Lake Michigan und Lake Erie, 
die Getreidetransporte von den Häfen der obern zu den unteren Seen und die Kohlentransporte von Lake Erie sowohl zu den obern Häfen wie auch zum Lake Ontario zu denken. Diese Bedeutung führte dazu, daß vorerst alle die Seen unter sich verbindenden Wasserstraßen, welche für die großen, diese Massentransporte ausführenden Schiffe zu klein wurden, ausgebaut wurden. Hingegen blieb vorerst die Verbindung om Lake Ontario zum offenen Ozean von diesen Ausbauplänen unberührt. Ein Glied in diesem System von Binnenwasserstraßen bildete der verbesserte Welland-Kanal (1913-1932) mit jetzt nur noch 8 Schleusen und 7,6 bis 8,25 m Tiefe. Weitere Glieder betrafen die verbesserten Fahrrinnen im Detroit und St. Clair River sowie die gewaltige Schleusenanlage bei Sault Sainte Marie. Es ergibt sich daraus, daß nach 1932 im wesentlichen nur noch der Abschnitt Lake Ontario bis Montreal auszubauen war, um eine für große Ozeandampfer befahrbare Wasserstraße von den Great Lakes zum Atlantik zu besitzen. Wenn man vom St. J-awrence Seaway spricht, versteht man darunter vom gesamten Wasserstraßennetz nur diesen Sektor». Die bis dahin errichteten Kanäle erlaubten kleineren Ozeandampfern mit Abmessungen von 77,4, 13,29 und 4,27 m, den sogenannten «canalern», vom Atlantik zu den Großen Seen zu gelangen, was bereits seit $1930 \mathrm{zu}$ einem regelmäßigen Linienverkehr geführt hatte. Er nahm namentlich seit dem $Z$ weiten Weltkrieg zusehends zu und erreichte 1958 über 400 sogenannte «round trips», an welchen mehrere europäische Schiffahrtsunternehmen beteiligt waren. Dies und der wachsende Bedarf an Energie namentlich im südlichen Kanada ließ endlich das endgültige, auf Grund schon zu Beginn des 20. Jahrhunderts unternommener Vorstudien konzipierte Projekt reifen.

Die Ausführung leitete auf kanadischer Seite die St. Laurence Seaway Authority, auf amerikanischer die St. Lawrence Seaway Development Corporation, wobei die schon 1909 gegründete International Joint Commission die Arbeiten zu koordinieren und zu überwachen hatte. Die Kraftwerksarbeiten waren der Hydro-Electric Power Commission of Ontario und der Power Authority of New Y'ork State anvertraut. Alle Unternehmungen arbeiteten in engem Kontakt miteinander, was erlaubte, das Werk wie geplant rechtzeitig zu Beginn 1959 abzuschließen. Als Kombination von Kanälen und Kraftwerken stellt es zweifellos eine hervorragende Leistung dar. Sie ist in fünf sogenannte Sections gegliedert: in die Thousand Island Section, eine Fahrrinne auf Seen und im obern Stromabschnitt, die kanalisierte International Rapids Section zwischen Prescott und Cornwall, die Lake St. Francis Section zwischen Cornwall und Bellerive (mit Schleusen versehene Strecken des alten Kanalsystems), die Soulanges Section (neuer mit Schleusen ausgestatteter Kanalabschnitt) und die Lake St. Louis Section (mit 5 Kraftwerken und 6 Dämmen), bildet es ein gewaltiges System von Anlagen, das naturgemäß auch die Landschaft bemerkenswert umgeformt hat.

Bereits 1932 hatte man als Oberwasser des Kraftwerkes Beauharnois (Staustufe $25,6 \mathrm{~m}$ ) auf kanadischem Boden den Beauharnois-Kanal auf 8,25 m Fahrtiefe ausgeweitet, und 1954-1958 wurde auf der Grenze zwischen Kanada und USA ein Grenzkraftwerk als zweite höhere Stufe ausgebaut. Sodann errichtete Kanada westlich von Cornwall unter Einbeziehung einiger Strominseln (Barnhart Inseln) eine Reihe von Staudämmen, die eine Staustufe von insgesamt 27,4 m erzeugten. Der St. Lorenz wurde dadurch zu einem $50 \mathrm{~km}$ langen See erweitert, dessen Oberfläche mit 73,15 m nahezu das Niveau des Ontario Sees besitzt, so daß ein bei Iroquois angelegter Ausgleichsdamm nötig wurde. Das 1958 in Betrieb genommene Werk, das im Endausbau (1960), 1,9 Mio Kw erzeugen wird, gehört zur einen Hälfte (Robert Saunders St. Lawrence Generating Station) Kanada, zur andern (Robert Moses Power Dam) den Amerikanern, wobei indes jedem der beiden Partner $50 \%$ der gewonnenen Energie zuerkannt ist. Den Seeweg als solchen kennzeichnen, vom Ontario See her gesehen folgende Teilstücke: Den Regulierdamm bei Iroquois umgeht ein Schleusenkanal, der ganz auf kanadischem Gebiet verläuft. Unterhalb des Dammes benutzt die Schiffahrt 
den neuen Stausee als Fahrrinne. Die Staustufe bei Cornwall umfährt auf amerikanischer Seite ein Kanal mit zwei Schleusen (der Eisenhower- und der Snell-Schleuse), wobei seit dem Bau beide Ufer Kanada zugesprochen sind. Im folgenden Flußabschnitt wird eine weitere Verbreiterung des St. Lorenz, der Lake St. Francis, als Verkehrsweg verwendet. An dessen unterem Ende, bei Belleville-Walleyfiled, sind regulierende Stauwehre angebracht, die zugleich die Einfahrt zum bisherigen Soulanges-Kanal und zum Beauharnois-Kanal kontrollieren; vom Lake St. Francis aus führt die Fahrt durch den Beauharnois-Kanal, um mittelst zweier Schleusen bei Beauharnois den Lake St. Louis zu erreichen. Im untersten Abschnitt zwischen Lake St. Louis und Montreal sind ebenfalls starke Verlagerungen der frühern Kanalführung eingetreten. Im Gegensatz zum Lachine-Kanal, welcher die gleichnamigen Stromschnellen umgangen und dabei das Stadt- und Industriegebiet von Montreal durchquert hatte, zieht nun die Fahrrinne dem südlichen Ufer des St. Lorenz in einem neuen Kanal mit zwei Schleusen (Saint Catherines, Lambert) entlang, womit der erstgenannte mit dem SoulangesKanal außer Betrieb gekommen sind.

Übersicht ïber das Große-Seen-St. Lorenz-Schiffahrls-System vor und nach dem Bau

\begin{tabular}{|c|c|c|c|c|c|c|c|}
\hline \multirow[t]{2}{*}{ Kanalstrecken } & \multirow[t]{2}{*}{ L.ängen $\mathrm{km}$} & \multicolumn{2}{|c|}{$\underset{\text { Alter }}{\text { Tiefen m }}$} & Neuer & $\begin{array}{l}\text { Zahl d } \\
\text { Alter }\end{array}$ & $\begin{array}{l}\text { thleusen } \\
\text { Neuer }\end{array}$ & \multirow[t]{2}{*}{ Größe m } \\
\hline & & Minimum & Seeweg & & & & \\
\hline St Mary's River & 100.8 & 6.3 & 7.5 & - & 0 & 0 & - \\
\hline Mac Arthur Schleuse & & & & & & & \\
\hline bei Sault Ste Marie & - & 9.3 & 9.3 & - & 1 & 0 & $243 \times 24 \times 9$ \\
\hline Straße von Mackinac & 32.0 & 8.1 & 8.1 & - & 0 & 0 & - \\
\hline St Clair u. Detroit River & 140.8 & 6.3 & 7.5 & - & 0 & 0 & - \\
\hline Welland Kanal & 44.8 & 7.5 & 7.5 & 9.4 & 8 & 0 & $243 \times 24 \times 9$ \\
\hline Thousand Islands & 108.8 & 8.1 & 8.1 & 9.4 & 0 & 0 & - \\
\hline International Rapids & 73.6 & 4.2 & 4.2 & 9.4 & 12 & 3 & $77 \times 13 \times 4$ \\
\hline Kanadische Sektion & 108.8 & 4.2 & 4.2 & 9.4 & 10 & 4 & $77 \times 13 \times 4$ \\
\hline Montreal bis Atlantik & 1600.0 & 10.5 & 10.5 & - & 0 & 0 & - \\
\hline
\end{tabular}

Daß beim ganzen Bauwerk Brücken für Straßen und Bahnen eine große Rolle spielten, braucht kaum gesagt zu werden. Um reibungslosen Verkehr zu gewährleisten, bestimmen die Bauvorschriften einheitliche minimale lichte Höhen unter den Brücken von $36,6 \mathrm{~m}$ wobei die bisherigen Dreh- und Schwenkbrücken der Eisenbahnen, die kurze Auffahrtsrampen hatten, zu Hebebrücken umgebaut wurden bzw. werden sollen, während Straßenbrücken in den meisten Fällen als hohe Bogenbrücken über Strom und Kanäle geführt werden. Gesamthaft gesehen ist wohl am bemerkenswertesten, daß die Erneuerung des Schiffahrtsweges 31 kleine durch 7 große Schleusen von 9,4 m Tiefe und 267,2 $\mathrm{m}$ Länge ersetzt hat, wodurch der Verkehr unzweifelhaft eine wesentliche Erleichterung erfuhr. Doch zeigten sich schon in den allerersten Tagen nach der Eröffnung technische Unzulänglichkeiten, die zu kleinern Unfällen und Verkehrsstauungen führten. Es wurde deshalb naturgemäß besonders von Seite der frühern Gegner des Projektes teilweise scharfe Kritik laut. Die größten Schwierigkeiten bereitete der nördliche Eingang zum Wellandkanal zwischen Ontario- und Erie See, wo oft bis zu 40 Schiffe mehrere Tage hindurch auf Abfertigung warten, ja sogar sich Reduktionen des Schiffsrumpfes gefallen lassen mußten. Die kanadische Regierung gab denn auch im Herbst 1959 bekannt, daß sie unverzüglich Verbesserungen am Kanal vornehmen lassen werde, welche seine Kapazität um rund $25 \%$ steigern sollen. Es verbleiben demgemäß wohl auch für die Zukunft noch verschiedene Aufgaben.

Die Gesamtkosten des Baues beliefen sich für den Seeweg auf 442 Millionen, mit den Bauten am Welland-Kanal auf 471 Millionen Dollar. An sie haben die USA 131 Millionen beizutragen. Die jährlichen Zukunftsaufwendungen für Betrieb, Unterhalt inkl. Zinsen und Amortisationen werden auf 28 Millionen Dollar geschätzt, 
wovon 25 Millionen auf den eigentlichen Seeweg entfallen sollen. Die zur Tilgung dieser Summen erhobenen Gebühren, die Kanada zustehen, sollen im Verhältnis 71 zu 29 zwischen diesem und den USA geteilt werden. Dabei werden für die Passage Erie-See-Montreal eine Grundgebühr von 6 Cents pro Registertonne plus 42 Cents je short-Tonne für Massengüter (Getreide, Mahlprodukte, Erze, Tankflüssigkeiten, Holz, Schrott usw.) und 95 Cents bei «general cargo» verlangt.

Den mutmaßlich-künftigen Güterverkehr zu beurteilen, dürfte nicht einfach sein. Nach den ursprünglichen amtlichen kanadischen und amerikanischen Schätzungen soll er von den 13 Millionen Tonnen im Jahre 1958 bis 1965 auf rund 50 Millionen gesteigert werden. Jedoch betrachten verschiedene Fachleute dies als überhöhte Veranschlagung. Im einzelnen sehen die Schätzungen folgende Zunahme (in Millionen Tonnen) voraus:

Güter

Allgemeinfrachten

Kohlen und Koks

Getreide

Eisenerz

Nichteisenerze

Erdöl und Erdölprodukte

Holzstoff

Total

$\begin{array}{ccccc}\begin{array}{c}\text { Mittel } \\ 1950-1954\end{array} & \begin{array}{c}\text { St. Lawrence Seaway } \\ \text { Development Corporation }\end{array} & \begin{array}{c}\text { Canadian } \\ \text { Department of Trade } \\ \text { and Commerce }\end{array} & \begin{array}{c}\text { Great Lakes } \\ \text { St. Lawrence } \\ \text { Association }\end{array} \\ 1362 & 6400 & - & 9876 & 5700 \\ 1646 & 3700 & - & 4200 & 6000 \\ 3481 & 12100 & - & 8200 & 10000 \\ 216 & 10500 & - & 20000 & 20000 \\ 77 & 800 & - & - & 1000 \\ 1547 & 2300 & - & 1091 & 2000 \\ 550 & 700 & - & 1165 & 1000 \\ 8879 & 36500 & 52000 & 44532 & 45700\end{array}$

Von den Massengütern wird dem Wasserweg namentlich der Getreidetransport aus dem kanadischen und amerikanischen Mittelwesten (Prärieprovinzen) zum Vorteil gereichen. Denn bei ihm kann mit einer erheblichen Frachtermäßigung durch den Einsatz größerer Schiffe und die Ausschaltung des zeitraubenden Umlads in Montreal gerechnet werden. Mindestens ebenso große Wichtigkeit wird, wie schon anfangs erwähnt, die Beförderung von Eisenerzen aus Labrador und Nordquebec nach den Stahlwerken von Cleveland, Chicago, und Detroit gewinnen. Beide Frachtgruppen dürften voraussichtlich $80-90 \%$ des gesamten Güterverkehrs beanspruchen. Für die amerikanische Stahlindustrie ist die verbilligte $Z$ ufuhr von Erzen von umso größerer Bedeutung, als die bedeutenden Vorkommen der Mesabi-Range am Lake Superior der Erschöpfung nahe scheinen, wodurch die binnenländischen Schwerindustriegebiete sich der Gefahr ausgesetzt sehen, durch Verlagerung von Betrieben an den St. Lorenz oder an die atlantische Küste entscheidender Wirtschaftsgrundlagen verlustig zu gehen.

Wie weit sich das Ausland am Schiffsverkehr auf der neuen Wasserstraße beteiligen wird, erscheint vorerst noch als ungelöste Frage. Noch 1958 fuhren von den den alten Kanal passierenden 4696 Handelsschiffen 3911 oder mehr als 93\% unter kanadischer Flagge; der Anteil der ausländischen Boote war somit gering, wobei die deutschen mit rund zwei Fünfteln vor den norwegischen, englischen, schwedischen, holländischen und dänischen durchaus dominierten. Außerdem fällt das Fehlen amerikanischer Schiffe auf, die auch 1959 zu konstatieren war. Der Grund liegt in der Subventionswirtschaft der amerikanischen Handelsschiffahrt, die infolge hoher Betriebskosten ohne Subventionen nicht zu existieren vermag, bis vor kurzem aber solche für den St. Lorenzseeweg nicht erhielt. Das Jahr 1959 hat indessen bereits eine erhebliche Zunahme fremder Schiffe gezeitigt, was nicht zuletzt aus den Reklamationen ausländischer Reedereien bei den anfänglichen Stauungen der Schiffahrt ersichtlich war.

Auch hinsichtlich der Auswirkungen der Schiffahrtsstraße auf die Wirtschaft der Anliegergebiete sind die Ansichten noch geteilt. Zweifelsohne dürfte die Industrie dieser Regionen einen Aufschwung erfahren, wenn auch die von der Propaganda in die Diskussion gerückten Vorteile kaum so groß sein werden wie dies geschildert wurde. Doch erhofft man von der Möglichkeit des verbilligten Bezuges verschiedener 


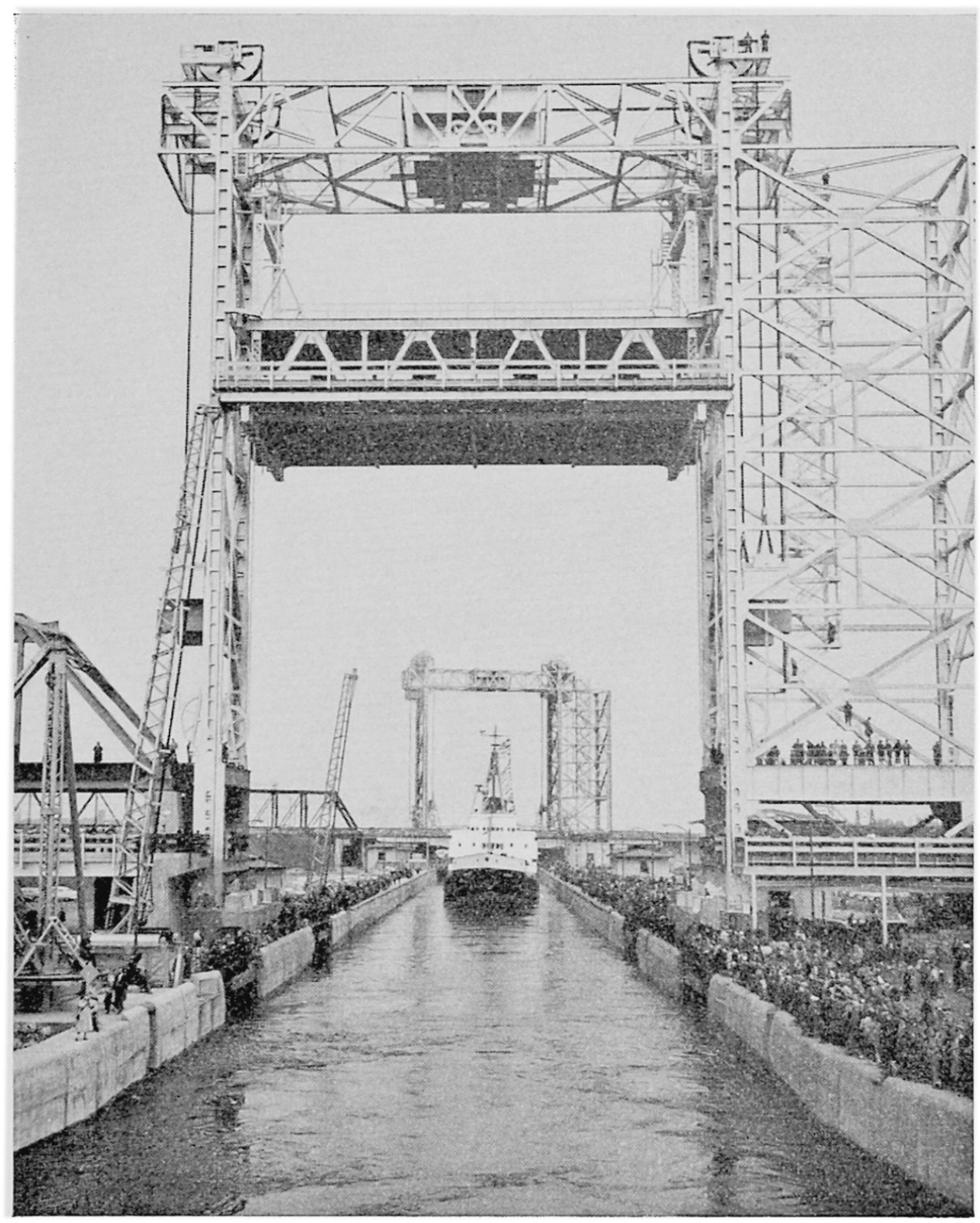

Die St. Lambock-Schleuse am 26. Juni 1959. Die Jacht der englischen Königin durchfährt den Schleusenkanal anläßlich der Einweihung des St. Lorenz-Seeweges.

Rohstoffe eine Vermehrung verschiedener Industrieunternehmungen, so etwa der Zukkerraffinerien und Kaffeeröstereien um Chicago und andernorts. Zudem werden aus dem verbilligten Güterverkehr namentlich die Exportindustrien wie die Autobranche und die Industrien landwirtschaftlicher Maschinen profitieren, obgleich umgekehrt hieraus auch die ausländische Konkurrenz Nutzen ziehen wird. Wenn in diesem Zusammenhang für den wichtigen Umschlagshafen Montreal ernste Befürchtungen laut geworden sind, die darauf hinweisen zu müssen glaubten, daß ihm der Umlad der Güter (Getreide) von den canalern auf die Ozeandampfer verloren gehen werde, so scheint sich diese Prophezeiung kaum zu bewahrheiten. Die Vorteile, die auch Montreal von der Verbesserung des Seeweges erhofft, sollen allen Mutmassungen nach die Nachteile durchaus überwiegen. Sonst hätte wohl kürzlich kaum der National Harbours Board einen Kredit von 57 Millionen Dollar für den Ausbau seines Hafens, vor allem auch für neue Getreidesilos bewilligt. Daß übrigens auch hier mit einer verstärkten Industrialisierung zu rechnen ist, beweist die Entstehung neuer Fabriken auf der Südseite des St. Lorenz. Sie wird sicher noch gefördert werden, wenn die Provinzregierung von 
Quebec sich entschließt, die Ausnützung der nahegelegenen Lachine Rapids, die nach Vorprojekten mindestens eine Million $\mathrm{kW}$ billiger Energie liefern können, in die Wege zu leiten.

Durch die Kanal- und Kraftwerkanlagen wurden übrigens rund 8000 Hektaren kanadischen und 7200 Hektaren amerikanischen Agrarlandes beansprucht. Dadurch mußten 6500 Kanadier der Dörfer Iroquois, Aultsville, Farran's Point, Dickinsons's Landing, Wales, Moulinette, Mille Roches und ein Drittel der Stadt Morrisburg, insgesamt 360 Farmen, in den USA 225 Farmfamilien und 500 Eigentümer von Sommersitzen umgesiedelt werden. Dazu kam die Verlagerung von 18 Friedhöfen, zahlreichen historischen Denkmälern sowie Straßen- und Eisenbahnstrecken, Elektrizitäts- und Telefonleitungen, was teilweise zu großen Besorgnisssen bei der Bevölkerung Anla $\beta$ gab. Bisher entstanden drei neue Gemeinden: Iroquois, Ingleside und Long Sault 3-20 km entfernt von den alten Siedlungen. Auch die ländliche Siedlungslandschaft erhielt also bereits während des Seewegbaues neue $Z$ üge.

Wenn man die zu erwartenden Einflüsse des neuen Seeweges auf Wirtschaft und Landschaft der Region zu beurteilen versucht, sind die Hoffnungen nicht zu vergessen, die namentlich auf der amerikanischen Seite hinsichtlich einer bemerkenswerten Zunahme des Tourismus gehegt werden. Wenn bisher vornehmlich die Landschaft der «Tausend Inseln» bei Massena durch ihre Reize jährlich tausende von Besuchern anzog - was sich übrigens seit langem in zahlreichen Ferienhäusern, Hotels und Campingplätzen äußerte - wird nun erwartet, daß künftig vor allem auch die technischen Bauwerke: die Riesenschleusen, Kraftwerke und nicht zuletzt auch Dämme und Brücken in vermehrtem Maße Touristen anlocken werden. So rechnete man allein in Massena für 1959 eine Million Gäste und die Behörden suchten sich auf einen Massenbesuch mittelst der Anlage von Plattformen und Gallerien vorzubereiten. In der nähern und weitern Umgebung entstanden ebenfalls bereits 1959 zahlreiche Motels, und die zwischen der Eisenhower-Schleuse und dem Kraftwerk Mose-Saunders gelegene Barnhart-Insel steht im Begriff, zu einem Nationalpark mit Badestränden, Campingplätzen usw. umgewandelt zu werden. Was hier für einen relativ kleinen wenn auch besonders stark geformten Teil der Stromlandschaft gesagt wurde, ließe sich in mehr oder weniger abgewandeltem Maße auf die ganze Baustrecke anwenden. Dafür legen etwa Vorhaben verschiedener Stromreedereien zur Einrichtung von Touristenkreuzfahrten zwischen Montreal und Chicago beredetes Zeugnis ab. Die 'Tatsache, da $\beta$ in diesem $\mathrm{Zu}$ sammenhang allein in Montreal einige Tausend Hektar Land für Park- bzw. Erholungsanlagen gekauft wurden, zeigt weiterhin, wie sehr Erwartungen auf eine $\mathrm{Zu}$ nahme wirtschaftlicher Einrichtungen nicht nur, sondern vor allem auch daraus resultierender Rekreationsbedürfnisse bestehen. Es scheint klar, daß im Gesamtrahmen besonders die Hafenstädte Vorteile aus einer Intensivierung des Verkehrs und Handels ziehen werden, wenn auch vorderhand noch zu viele sich an ihr zu beteiligen gedenken. Jedenfalls haben sowohl auf der amerikanischen als auf der kanadischen Seite des Stromes und der Seenfront eine größere Zahl bereits erhebliche Ausbauprogramme ihrer Häfen aufgestellt, so u. a. 'Toronto, Hamilton, Fort William und Port Arthur, Chicago, Gary, Milwaukee, Detroit, Duluth, Toledo, Cleveland, Buffalo, Oswego und Ogdensburg. Und in diesem Zusammenhang wird gewiß auch das Zusammenwachsen der Städte dieser Region, wird die Konurbation von «Mississaga», der in Verschmelzung begriffenen Städte Groß-Toronto, Groß-Hamilton, Groß-Windsor, der Niagara- und der Grand-River-Region, die bereits jetzt mehr als 2000000 Bewohner in sich schließt und für die - bei einem Gesamtareal von rund $10000 \mathrm{~km}^{2}$ - bis Ende des Jahrhunderts ein Anwachsen auf 5 Millionen prophezeit wird, gefördert werden. Die zuständigen Behörden haben also Grund, in Anbetracht dieser Aussichten, Land zu reservieren, das «grün» bleibt. Immerhin stehen einer allzu forcierten Entwicklung allerlei Hindernisse entgegen; von der bereits bestehenden Konkurrenz 


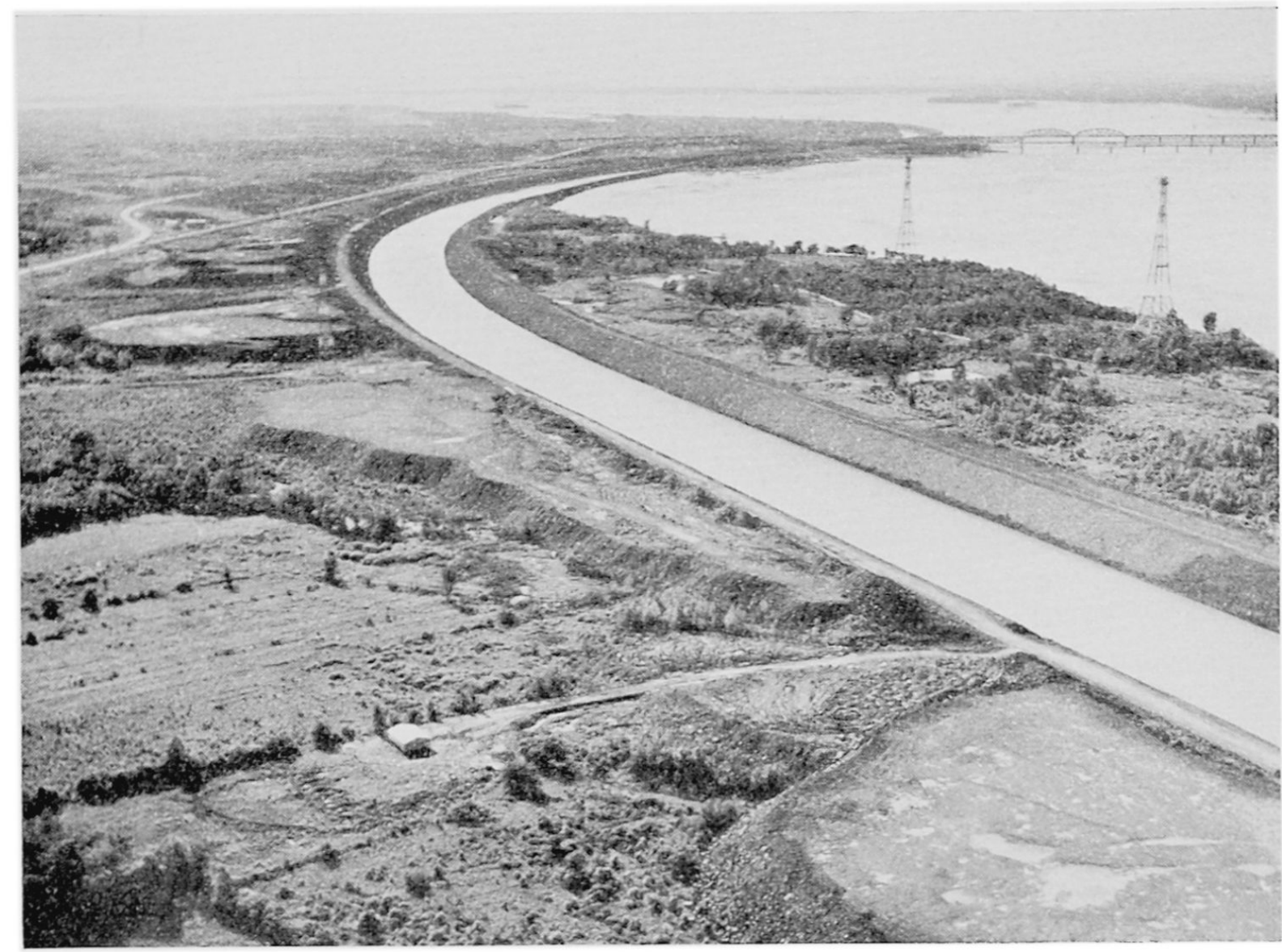

Kanallandschaft an der "Südküste» des St. Lorenz-Seeweges gegenüber Montreal. Der Kanal ist $16 \mathrm{~m}$ breit und $27 \mathrm{FuB}$ tief.

einer noch zu großen Anzahl von Häfen abgesehen sind es nicht zuletzt die Frachtstrukturen der Zubringerdienste (bes. der Bahngesellschaften), die durch die Vielzahl der Häfen gegebenen Zeitverluste bei der Frachtlöschung u. a., die vorläufig noch verzögernd wirken.

Die Weiterentwicklung der kandisch-amerikanischen Kulturlandschaft längs dem St. Lorenz und darüber hinaus sicher auch in seinem ganzen Einzugsgebiet hat nichtsdestoweniger einen Impuls erfahren, der sich über kurz oder lang auch in ihrem Antlitz noch nachdrücklicher ausprägen wird. Bereits zeigen die imposanten Kanal- und Kraftwerkbauten und ihre Annexeinrichtungen, daB der «herrliche Strom» ( $M$. PARDÉ) im Zuge ist, in noch vielfach verstärktem Maße zum Torweg (Gateway), zur Hauptstraße (Mainstreet) und zur «Schlagader» (Artery) namentlich Kanadas $\mathrm{zu}$ werden als er dies bereits bisher war. Umsomehr wird es klarer und sorgfältiger Landschaftsplanung und -weitergestaltung bedürfen, um das bisherige Werk zum dauernden Wohl des Landes werden zu lassen.

\section{HAUPTSÄCHLICH BEN ÜTZTE LITERATUR}

Wright, C. P.: The St. Lawrence Deep Waterway - A Canadian Appraisal. Toronto 1935. Blanchard, R.: L'Est du Canada Français. Montreal 1935; derselbe: Le Centre du Canada Français. Montreal 1947; derselbe: L’Ouest du Canada Français. Montreal 1951. - Pardé, M.: Hydrologie du Saint-I aurent et de ses affluents. Rev. Canad. de Géogr. 2, 1948, No 2 - 4. - Putwalı, D. F. (Editor): Canadian Regions. London 1952 (1958). - Bernhard, H. und Winkl.tr, E.: A Mari usque ad Mare. Bern 1953. - Belitzkr, A. H.: The St. Lawrence River Project. Syracuse 1954. - L'administration de la voie maritime du Saint-Laurent: La Voie Maritime du Saint-Laurent. Ottawa 1955. - Wood, H. A.: Recreational Land Use Planning in the St. Lawrence Seaways Area, Ontario. Community Planning 
Rev. 5, 1955, N.1; derselbe: The St. Lawrence Seaway and Urban Geography. Cornwall-Cardinal, Ontario. The Geogr. Rev. 45, 1955, N. 4; derselbe: The St. Lawrence Seaway and agricultural geography in the Cornwall-Cardinal area, Ontario. Geogr. Bulletin 8, 1956, Nr. 2-3. - W INKLER, E.: Kanadische New Towns. Plan 12, 1955, Nr. 11/12. - Hartley, H. R.: The Effects of the St. Lawrence Seaway on Grain Movements. Bloomington 1957. - Montreal Research Council, School of Commerce, McGill University: The Impact of the St. Lawrence Seaway on the Montreal Area. Montreal 1958. - BoEsch, H.: Die neue Wasserstraße in den nordamerikanischen Kontinent. Neue Zürcher Zeitung 180, 1959, Nr. 722. - HöffDing, W.: Die St. Lorenz-Wasserstraße. Neue Zürcher Zeitung 180, 1959, Nr. 1942, 2013. - Scotr, V. Taming the St. Lawrence. Discovery 20, 1959, Nr 6; Hills, T. L.: The St. Lawrence Seaway. London 1959. - Survey markets-Canada 1959. Montreal 1959. - Vgl. Canada Department of Mines and Technical Surveys, Geogr. Branch. Bibliographical Series. Ottawa $1950 \mathrm{ff}$.

\title{
THE ST. LAWRENCE SEAWAY
}

In the early summer 1959 the new St. Lawrence Seaway was opened, making the Great Lakes of North America accessible to the large ocean-going vessels of the world's seaports. After many decades of discussion between the USA and Canada work was finally begun on this enormous project and it was brought to completion in less than five years. The work consisted mainly in dredgeing out the waterway to a uniform depth of 10 yards and in the construction of new canals, particularly between the Lake Ontario and Montreal. Furthermore, the 31 smaller locks of the old waterway have been replaced by seven large locks. In addition, a vast hydro-electric power complex was built which will develop up to 1900000 kilowatts of electricity. The project required a large number of changes in the landscape, which probably will become even more prominent in future. For the economic life and culture of both neighbouring states, Canada and the USA, the «Main Street of North America» represents not only a vital line of communication but a symbol of international solidarity.

Der Verfasser dankt der Kanadischen Gesandtschaft in der Schweiz, Bern, bestens für die freundliche Überlassung von Photos aus dem National Film Board in Ottawa.

\section{LANGADI, ATYA-PATYA, SOWIE EINIGE VERWANDTE SPIELE DES FERNEN OSTENS*}

\author{
Paul G. Brewster
}

Eines der volkstümlichsten der in Bombay und Umgebung geübten Spiele ist Langadi, an welchem in der Regel nur Knaben teilnehmen. In diesem Fang-Spiel gelten die folgenden Hauptregeln:

1. Als Spielfeld dient ein Kreis von dreißig Fuß im Durchmesser. Innerhalb des Kreises befinden sich die Läufer und außerhalb desselben die Jäger. Sobald der Spielleiter pfeift, tritt einer der Jäger, auf einem Fuße hüpfend, in den Kreis und berührt so viele der Läufer als er vermag. Jeder, der vom Jäger berührt wurde, muß den Kreis sofort verlassen.

2. Es ist dem Jäger unter keinen Umständen erlaubt, beide Füsse auf den Boden zu setzen oder mit dem andern Fusse weiterzuhüpfen. So muß er, wenn er auf dem rechten $F u ß z u$ hüpfen begonnen hat, auf demselben weiterhüpfen, bis er erschöpft ist, oder bis aus einem anderen Grund der nächste Jäger seinen Platz einnehmen will. Verliert der Jäger das Gleichgewicht, so darf er sich dadurch zu helfen suchen, daß er beide Handflächen auf den Boden stützt. Wenn der Jäger nach dem Berühren eines Läufers das Gleichgewicht verliert und den andern Fuß auf den Boden setzt, so muß dieser Läufer den Kreis nicht verlassen. Kein anderer Körperteil des Jägers außer dem einen $\mathrm{Fu} \beta$ und den beiden Handflächen dürfen den Boden berühren, im andern Falle ist der nächste Jäger an der Reihe.

* Die Beschreibung der indischen Spiele verdanke ich der Freundlichkeit von Frl. Jaya S. Patel, Bombay, die Übersetzung ins Deutsche Hrn. dipl. Geogr. Arthur Dürst, Zürich. 